\title{
Cartographic Styles Used in Spatial Planning Maps to Visualise Uncertain, Unfinished and Imagined Content
}

\author{
Katharina Henggeler ${ }^{\mathrm{a}, *}$, Lorenz Hurni ${ }^{\mathrm{a}}$ \\ a Institute of Cartography and Geoinformation, ETH Zurich, \{khenggel|lhurni\}@ethz.ch \\ * Corresponding author
}

Keywords: Cartographic Style, Spatial Design, Uncertainty Visualisation, Fuzziness Visualisation, Map Cognition

\begin{abstract}
:
Traditionally, maps aspire to be as precise, clear, and unambiguous as possible. In spatial planning, however, the opposite is needed sometimes. Spatial planners increasingly have to deal with complex, multi-layered, and interdisciplinary problems. Often, there exists no 'main problem' to start with. Instead, planners are confronted with a heap of unsolved conflicts and tasks with unclear priority (see e.g., Figure 1). Such tasks can range from resolving the everyday traffic jam at a certain spot in the city to planning a new railway through a valley or ensuring enough affordable housing in an entire region for the next 15 years. During the creative process of finding solutions for such problems, planners create maps. The purpose thereby is manyfold: To get an overview of the situation; to play with bold or seemingly impossible ideas; to gain new insights; or to test, communicate, and preserve ideas, hypotheses, and concepts.
\end{abstract}

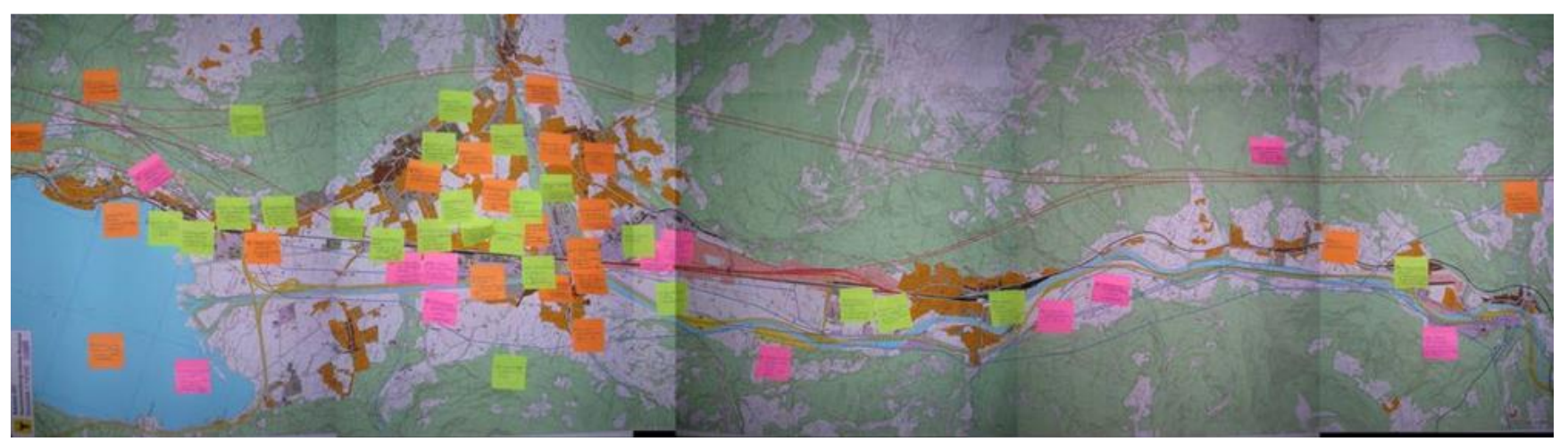

Figure 1. Unsolved conflicts and tasks as seen by the canton of Uri (Switzerland) at the beginning of the case study ('Testplanung') in 2006.

As a result, these maps do not only show 'finished' ideas and completely developed concepts. Instead, they often visualise 'partly-baked ideas'. That is, assumptions, hypotheses, suggestions, and questions, that are still open to discussion or, in other words, are uncertain (Figure 2 shows two examples of such maps). Showing this uncertainty often without explicitly quantifying it - is crucial for the creative design process. It leaves room for discussion and opens space for new ideas. 

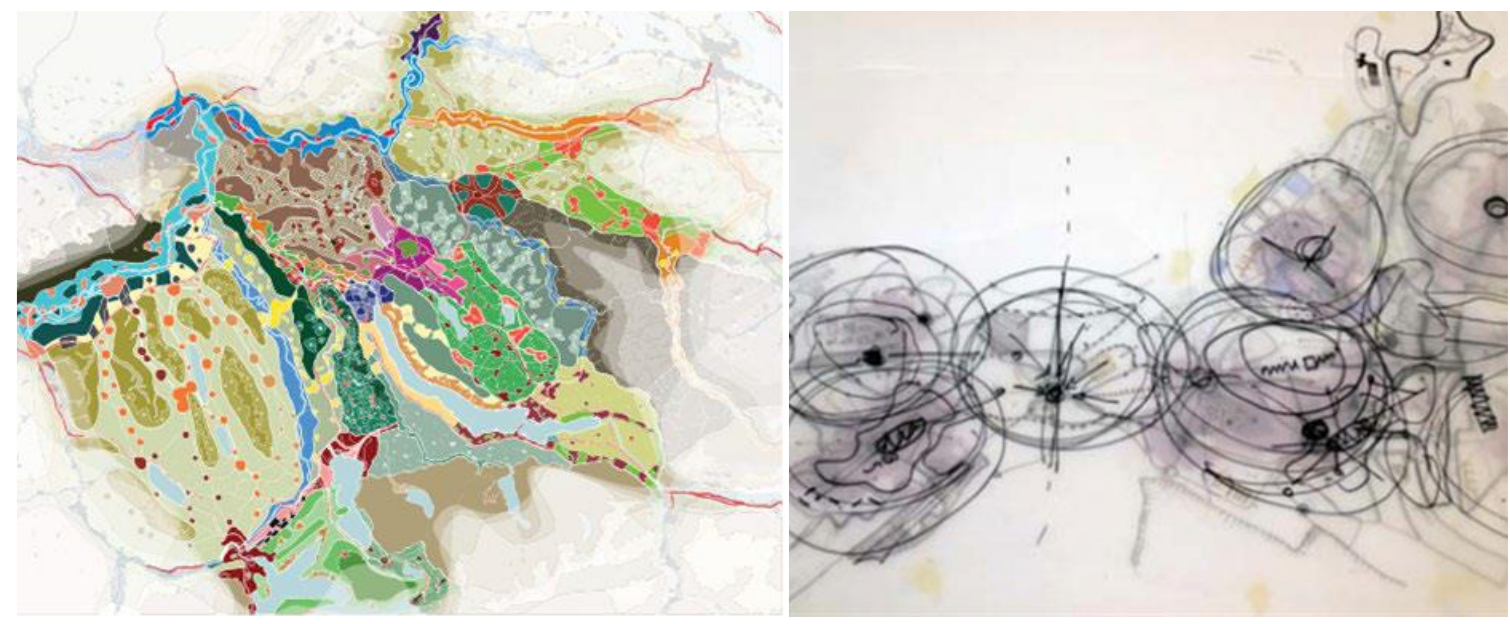

Figure 2: Exemplary spatial planning maps featuring uncertain content: Area types of unclear extent (left) and a suggestion of how to read a city's neighbourhoods (right).

But how exactly should these partly-baked ideas be visualised, especially their uncertain nature? Are there different types of uncertainty that need to be considered and what are they? In GIScience and cartography, there exists extensive research about different types of uncertainty and how to visually deal with them: for example, how to visualise objects with unclear boundaries or ways to automatically smoothen geometries. However, little research can be found about how they all come together in maps created during the spatial design process. Moreover, the aim of these proposed techniques usually remains to visualise the uncertainty in an as precise and unambiguous manner as possible, effectively reducing the uncertainty. As a result, there exist few guidelines about how such partly-baked ideas, and especially their uncertain nature, should be visualised in a spatial planning context.

A first step to finding an answer to these questions is to look at existing maps from the spatial design processes (such as, e.g., shown in Figure 2). We will give an overview of such maps, the types of uncertainty they feature, as well as the cartographic styles used to visualise these uncertainties. Different methods to derive this overview resp. categorisation, e.g., stylistic parameters, automatic classification through machine learning, manual approaches, will be presented. Such an overview will help understand the needs spatial planners have regarding the maps they create. 